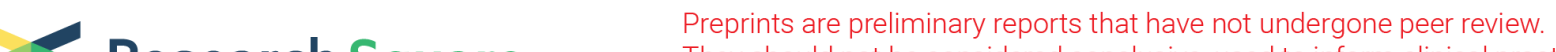 Research Square They should not be considered conclusive, used to inform clinical practice, or referenced by the media as validated information.
}

\section{Risk Factors Analysis of COVID-19 Patients with ARDS and Prediction Based on Machine Learning}

\section{Wan Xu}

Hangzhou Xiaoshan District Center for Disease Control and Prevention, Hangzhou, China

\section{Nan-Nan Sun}

Hangzhou Wowjoy Information Technology Co., Ltd, Hangzhou, China

\section{Hai-Nv Gao}

Department of Infectious Diseases, ShuLan (Hangzhou) Hospital Affiliated to Zhejiang Shuren University Shulan International Medical College, Hangzhou, China

\section{Zhi-Yuan Chen}

Hangzhou Wowjoy Information Technology Co., Ltd, Hangzhou, China

\section{Ya Yang}

Bin Ju ( $\sim$ bin.ju@wowjoy.com )

Hangzhou Wowjoy Information Technology Co., Ltd, Hangzhou, China

\section{Ling-Ling Tang ( $\sim$ lingling.tang@shulan.com )}

Department of Infectious Diseases, ShuLan (Hangzhou) Hospital Affiliated to Zhejiang Shuren University Shulan International Medical College, Hangzhou, China

\section{Research Article}

Keywords: ARDS, COVID-19, Clinical features, Risk factors, Machine learning

Posted Date: January 4th, 2021

DOI: https://doi.org/10.21203/rs.3.rs-77820/v2

License: (9) This work is licensed under a Creative Commons Attribution 4.0 International License. Read Full License 


\section{Abstract}

COVID-19 is a newly emerging infectious disease, which is generally susceptible to human beings and has caused huge losses to people's health. Acute respiratory distress syndrome (ARDS) is one of the common clinical manifestations of severe COVID-19 and it is also responsible for the current shortage of ventilators worldwide. This study aims to analyze the clinical characteristics of COVID-19 ARDS patients and establish a diagnostic system based on artificial intelligence (AI) method to predict the probability of ARDS in COVID-19 patients. We collected clinical data of 659 COVID-19 patients from 11 regions in China. The clinical characteristics of the ARDS group and no-ARDS group of COVID-19 patients were elaborately compared and both traditional machine learning algorithms and deep learning-based method were used to build the prediction models. Results indicated that the median age of ARDS patients was 56.5 years old, which was significantly older than those with non-ARDS by 7.5 years. Male and patients with BMI $>25$ were more likely to develop ARDS. The clinical features of ARDS patients included cough (80.3\%), polypnea (59.2\%), lung consolidation (53.9\%), secondary bacterial infection (30.3\%), and comorbidities such as hypertension (48.7\%). Abnormal biochemical indicators such as lymphocyte count, CK, NLR, AST, LDH, and CRP were all strongly related to the aggravation of ARDS. Furthermore, through various Al methods for modeling and prediction effect evaluation based on the above risk factors, decision tree achieved the best AUC, accuracy, sensitivity and specificity in identifying the mild patients who were easy to develop ARDS, which undoubtedly helped to deliver proper care and optimize use of limited resources.

\section{Introduction}

The coronavirus disease 2019 (COVID-19) is an acute infectious pneumonia caused by a severe acute respiratory syndrome-coronavirus-2 (SARS-CoV-2) infection previously unknown to humans. Spreading mainly through the droplet route and close contact, the virus causes mild symptoms in the majority of cases, the most common being: fever, dry cough, and fatigue[1, 2].

The disease has the characteristics of fast transmission and strong infectivity[3]. Since the outbreak in early December 2019 in Wuhan, China, it has rapidly developed into a worldwide pandemic, with more than 3 million patients confirmed to have been diagnosed with the disease in more than 200 countries, and the number of infected people is probably much higher. As of April 30, 2020, 217769 people died of COVID-19 infection. Despite the public health responses aimed at containing the disease and delaying its spread; during the courses of treatment, due to the large increase in the demand for hospital beds and the shortage of medical equipment, coupled with the lack of specific medicine, patients with basic diseases or old age are more likely to progress to severe disease, leading to death. Recent reports show that 14.1$33.0 \%$ of COVID-19 infected patients are prone to develop into severe cases, and the mortality rate of critical cases is $61.5 \%$, increasing sharply with age and underlying comorbidities[4-7]. Furthermore, 
medical staff may also be infected, which makes many countries face critical care crisis. COVID-19 poses an important and urgent threat to global health.

Acute Respiratory Distress Syndrome (ARDS) is a common and devastating critical illness[8]. It has been reported that $67 \%$ of COVID-19 patients with the severe illness have developed ARDS, which is the main cause of death[9]. However, in the early stage of onset, quite a few patients have no obvious clinical symptoms, so it is difficult to judge until ARDS occurs. Predicting which patients are more likely to develop ARDS, and thus face a greater risk of complications including death is particularly important in a novel and accelerating outbreak[10]. It would be useful in evaluation or prediction the public health burden or resources demand in a large scale e.g. in a city or a province.

Artificial intelligence (Al) has begun to tackle these difficult challenges in healthcare and it can provide clinical decision support if used carefully[11]. Currently, the prediction models of COVID-19 reported mainly focus on epidemics trend, early screening, CT diagnosis, and prognosis of COVID-19 patients[1215]. Few models have been studied for early identification of patients who are most likely to develop ARDS and recommending interventions. Xiang Bai et al. established a Long Short-Term Memory (LSTM) model by combining 75 clinical features and a quantitative CT sequence data obtained at different times to predict the malignant progression of COVID-19, which achieved an AUC of 0.954[16]. Xiangao Jiang et al. used traditional machine learning methods such as decision tree(DT), random forest(RF), and support vector machine(SVM) to predict disease progression to ARDS in COVID-19 patients, with the overall accuracy of $70 \%-80 \%[10]$. This study was a small sample prediction model of only 53 patients, so the prediction accuracy was slightly lower. The most-reported predictors of severe progression in patients with COVID-19 included age, sex, features derived from computed tomography scans, $C$ reactive protein, lactic dehydrogenase, and lymphocyte count.C index of these models ranged from 0.85 to 0.98 [17]. However, most reports did not include a description of the study population or intended use of the models and were rated at high risk of bias at the same time. Early detection of patients who are likely to develop critical illness is of great importance and may help to deliver proper care and optimize use of limited resources. We aimed to develop the COVID-19 ARDS clinical decision support system using machine learning algorithms and deploy it into electronic medical records(EMR) to assist doctors in identifying severe patients at the time of hospital admission.

\section{Results}

\subsection{Characteristics of COVID-19 patients $\llbracket$}

Tables 1 to 3 lists the distribution of various parameters including demographic, epidemiology and clinical characteristics of the COVID-19 ARDS and non-ARDS populations.

- Demographics and Epidemiology

In this study, we collected a total of 659 patients from Wuhan and non-Wuhan areas who were confirmed with COVID-19囚of which 76 patients (11.5\%) developed ARDS. 447 patients (70.9\%) had contact with 
infected persons and $50.9 \%$ had a family infection. The median incubation period was 5 days (interquartile range, 3 to 9) and the average time from onset to ARDS and admission to ARDS were 10 days and 3 days, respectively. The median age of the patients was 50 years (interquartile range, 37 to 62 ) and $50.4 \%$ of the patients were male. Patients with ARDS were significantly older than those with nonARDS by a median of 7.5 years ( 56.5 years vs. 49 years) and male patients $(76.3 \%)$ were more likely to develop ARDS. More than $50 \%$ of ARDS patients had a BMI greater than 25 . However, the exposure histories of the two groups were similar (Table 1).

- Clinical Characteristics and Underlying Diseases

On severity evaluation at admission, $75.4 \%$ of COVID-19 patients were assessed as common type while among the patients with ARDS, $80.3 \%$ were evaluated as severe or critical. The most common clinical symptoms of COVID-19 patients at the time of onset were fever (66.6\%), cough (68.7\%), expectoration (39.6\%), fatigue (34.2\%) and dry cough (29.6\%). Encephalopathy (0.5\%), hemoptysis (1.6\%), vomiting (3.0\%) and stuffy nose (3.8\%) were uncommon. Compared with non-ARDS patients, ARDS patients had a higher frequency of coughing ( $80.3 \%$ vs. $67.2 \%)$ and dyspnea $(59.2 \%$ vs. $11.6 \%)$. The median temperature was $37.4^{\circ} \mathrm{C}$. ARDS patients were $0.5^{\circ} \mathrm{C}$ higher than non-ARDS patients $\left(37.9^{\circ} \mathrm{Cvs} .37 .4^{\circ} \mathrm{C}\right)$, which was statistically significant $(P<0.001)$.

Overall, the presence of any comorbidities was more common among ARDS patients than no-ARDS (56.6\% vs. 39.8\%). Patients with ARDS had a much higher incidence of hypertension ( $48.7 \%$ vs.23\%) and diabetes (17.8\% vs.9.5\%). Two of the five patients infected with other viruses developed ARDS. ARDS also occurred in one patient who was treated with immunosuppressive agents (Table 2).

- Radiologic, Laboratory Findings and Complications

Table 3 shows the results of radiologic, laboratory findings on admission and complications. $74.7 \%$ of the patients presented ground-glass shadows on chest CT images and $28.3 \%$ of the patients presented consolidation. The above two imaging features accounted for a higher proportion of patients with ARDS than non-ARDS patients, which were $80.8 \%$ vs $73.9 \%$ and $53.9 \%$ vs $24.7 \%$, respectively. The median number of consolidation in ARDS patients was two.

Within 48 hours of admission, lymphocytopenia was present in $23.4 \%$ of the patients and leukopenia in $24.8 \%$. However, among ARDS patients, $19.8 \%$ had an increase in the white blood cell count, which indicated that ARDS patients had a secondary infection. The ratio of neutrophils to lymphocytes was greater than 3 in $45.3 \%$ of COVID-19 patients and $82.7 \%$ in ARDS patients with a Median of $6.11 .47 .7 \%$ and $32.2 \%$ of patients had elevated levels of C-reactive protein and lactate dehydrogenase, respectively. In a small number of patients, levels of alanine aminotransferase (ALT), glutamate aminotransferase (AST), creatine kinase (CK) and D-dimer were elevated. Laboratory abnormalities were more severe in ARDS patients than in non-ARDS patients. Besides, the medians of myoglobin and fasting glucose in ARDS patients were $85.9 \mu \mathrm{g} / \mathrm{L}$ and $8.1 \mathrm{mmol} / \mathrm{L}$ respectively, which exceeded the normal reference range and was significantly different from the non-ARDS group. 
During hospitalization, $91.3 \%$ of patients were diagnosed with pneumonia, and there was no statistical difference between the ARDS group and non-ARDS group. However, patients with ARDS had a higher incidence of shock and secondary bacterial infection (5.5\% and 30.3\%) than those with non- ARDS ( 0 and $4.3 \%$ ), and $45.2 \%$ of them were admitted to ICU (Table 3 ).

\subsection{Prediction of risk factors for COVID-19 ARDS}

After removal of variables with missing rate $>20 \%$, a total of 98 variables consisting of demographic, epidemiology, clinical symptoms, underlying diseases, complication, CT image features and laboratory results were extracted from the structured and unstructured data of electronic medical record (EMR) according to literature reviews and expert clinician opinions. Then, we selected 19 significant risk factors related to COVID-19 by means of SPSS single factor analysis. Among all risk factors, severity evaluation at admission (odds ratio [OR], 13.206; 95\% Cl, 8.550-20.397; $\mathrm{P}<0.001)$, gender (OR, 3.312; 95\% Cl, 1.979$5.544 ; \mathrm{P}<0.001)$, age ( $\geq 70$ year) (OR⿴19.811; 95\%Cl, 4.473-87.741; $\mathrm{P}<0.001) \varangle \mathrm{BMI}(<23$ vs. $>25)$ (OR, 3.717; $95 \% \mathrm{Cl}, 1.966-7.062 ; \mathrm{P}<0.001)$, temperature $\left(>39^{\circ} \mathrm{C}\right)(\mathrm{OR}, 5.279 ; 95 \% \mathrm{Cl}, 2.305-12.090 ; \mathrm{P}<0.001)$, hemoptysis (OR, 7.307; 95\% Cl, 2.263-23.595; $\mathrm{P}<0.001)$, cough (OR, 2.574; 95\% Cl, 1.429-4.542; $\mathrm{P}<0.001)$, shortness of breath (OR, 11.281; 95\%Cl, 6.883-18.490; $\mathrm{P}<0.001)$, hypertension (OR, $4.105 ; 95 \% \mathrm{Cl}, 2.572-$ 6.554; $P<0.001)$, diabetes (OR, 2.176; 95\%Cl, 1.161-4.078; $P<0.001)$, secondary bacterial infection (OR, 9.686; 95\% Cl, 5.146-18.323; $\mathrm{P}<0.001$ ), lung consolidation (OR, 4.264; 95\% $\mathrm{Cl}, 2.668-6.815 ; \mathrm{P}<0.001)$, lymphocyte count $(\mathrm{OR}, 0.145 ; 95 \% \mathrm{Cl}, 0.080-0.263 ; \mathrm{P}<0.001)$, neutrophils/lymphocytes ratio (NLR) (<3 vs. 23) (OR, 7.211; 95\%Cl, 3.980-13.064; $\mathrm{P}<0.001), \mathrm{ALT}(\leq 40$ vs. $>40 \mathrm{U} / \mathrm{L})(\mathrm{OR}, 2.710 ; 95 \% \mathrm{Cl}, 1.639-4.482$; $\mathrm{P}<0.001), \mathrm{AST}(\leq 40$ vs. $>40 \mathrm{U} / \mathrm{L})(\mathrm{OR}, 5.139 ; 95 \% \mathrm{Cl}, 3.100-8.520 ; \mathrm{P}<0.001), \mathrm{CK}(\leq 185 \mathrm{vs} .>185 \mathrm{U} / \mathrm{L})(\mathrm{OR}$, 4.114; 95\% Cl, 2.312-7.319; $\mathrm{P}<0.001)$, lactate dehydrogenase (LDH) ( $\leq 250$ vs. $>250 \mathrm{U} / \mathrm{L})(\mathrm{OR}, 8.104$; 95\%Cl, 4.733-13.876; $\mathrm{P}<0.001)$, C-reactive protein (CRP) ( $\leq 10$ vs. $>10$ mg/L) $(\mathrm{OR}, 5.959 ; 95 \% \mathrm{Cl}, 3.510-$ 10.119; $\mathrm{P}<0.001)$ were all strongly correlated with ARDS (Table 4).

\subsection{Development and verification of predictive models}

Based on the above results of univariate analysis, we determined 19 risk factors including severity evaluation at admission, gender, age, BMI, temperature, cough, shortness of breath, hemoptysis, hypertension, diabetes, secondary bacterial infection, lung consolidation, lymphocyte count, CK, NLR, ALT, AST, LDH, and CRP as inputs to the model to evaluate whether COVID-19 patients would develop ARDS. We tried five algorithms for modeling, including logistic regression (LR), random forest (RF), support vector machine (SVM), decision tree (DT) and deep neural networks (DNN). Table 5 shows the mean \pm standard deviation (std.) for 10 -fold cross validation with AUC and accuracy. DTQLR and RF all exceeded AUC of 0.85 and the mean accuracy of each algorithm was over 0.8 . In order to further verify the accuracy of the models, performances of five algorithms were evaluated on the external test set with each technique. Table 6 and figure 1 show that DT, LR, RF and DNN all demonstrated good performance in term of AUC, accuracy and specificity. The sensitivity of DT and LR was much higher than that of other three models. Considering the unbalance of the actual dataset, we also evaluated the balanced accuracy of each model. The result of DT and DNN was 0.98 and 0.93 , respectively. The predictive model 
established by SVM exhibited the worst performance in five models. It is necessary for ARDS diagnosed tool with high sensitivity and accuracy. The results show that DT marked the best value in each evaluation with AUC of 0.99 , accuracy of 0.97 and sensitivity of 1.0 respectively. Therefore, the model constructed by decision tree algorithm was optimum tool for ARDS prediction.

\section{Discussion}

In this study, we comprehensively compared the clinical characteristics of all confirmed COVID-19 patients with and without ARDS『and determined 19 features for modeling. All included variables were strongly correlated with disease progression. Age ( $>70$ years), gender, hypertension, diabetes as well as severity evaluation are recognized risk factor for developing ARDS in COVID-19 patients [18]. Clinical manifestations such as fever, cough, hemoptysis, shortness of breath and lung consolidation reflect the progression of COVID-19[19-20]. Viral infections predispose patients to secondary bacterial infections, which often lead to a more severe clinical course. Secondary bacterial infection has been considered as a critical risk factor for the severity and mortality rates of COVID-19 despite antimicrobial therapies [21,22]. Lymphopenia, high concentrations of CRP and LDH may indicate severe acute lung inflammatory reaction and cell damage [23-25], which has been reported to be risk factors for severe patients with COVID-19 [26]. ALT and AST are markers of acute liver injury. Studies have found that abnormal liver tests in patients with COVID-19 were associated with the progression to severe pneumonia. The detrimental effects on liver were mainly related to the use of lopinavir/ritonavir during hospitalization. Therefore, liver function should be monitored and evaluated frequently during medication $[27,28]$. NLR is an indicator of systemic inflammation [29], mainly seen in tumor-related diseases, autoimmune diseases, bacterial infectious pneumonia and tuberculosis [30-33]. It was reported that COVID-19 infection-triggered inflammation increased NLR, which was significantly associated with poor clinical outcomes of COVID-19 patients [34]. We found that CK was a high-risk factor for ARDS. On the one hand, it might be associated with heart injury in critically ill patients with COVID-19 [35]. On the other hand, this indicator was related to rhabdomyolysis [36-37].Several cases of rhabdomyolysis were reported in COVID-19 severe patients, with a marked increase of CK [38-40].

We tried five algorithms for modeling and finally the decision trees performed best. In clinical prediction research, decision tree is frequently designed to build binary classifiers, such as cancer prediction/prognosis [41]. As a method used in machine learning, it is nonparametric which makes fewer data assumptions and it can accommodate collinear independent variables [42]. It is also less sensitive to outliers and more robust to high-dimensional data, which possess many independent variables relative to outcomes [43]. The main advantage of decision tree is its simple structure, which allows for better extracting classification rules and interpretation. Our model consisted of 19 clinical variables, which were all relatively inexpensive and easy to be obtained directly from clinical symptoms and routine laboratory tests. At the same time, the system showed good sensitivity, specificity and AUC in the external test cohort. Compared with the results of Jiang et al.[10], the overall accuracy of our model is higher (70\% vs $91 \%)$. 
Our study has several strengths: first, we have successfully used a machine learning algorithm to analyze clinical datasets and developed a diagnosis aid system, which has been deployed in electronic medical records for early identification of ARDS in COVID-19 patients. By submitting clinical information online, medical staff can triage patients at hospital admission based on the predicted risk factors and arrange patient treatment plans accordingly, ensuring patients receive treatment early and medical resources can be efficiently allocated. Secondly, to ensure the reliability of the conclusion, we used data from multicenters with large samples for modeling and verification. Third, we found that CK (>185 U/L) and NLR were strongly correlated with ARDS, which might be the new potential early identification biomarkers in COVID-19 severe patients.

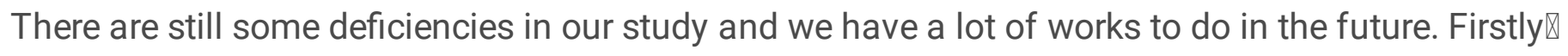
although we collected data of 659 COVID-19 patients in multiple centers, samples available for ARDS were limited. Secondly, we did not collect CT images data, and the quantitative information of CT diagnostic data was not detailed enough. Thirdly囚it has been reported that D-dimer was a risk factor for COVID-19 severity. However, due to a large number of missing data, similar conclusions were not reached in our study. Finally, it is of great clinical value to study the intervention measures and prognosis of COVID-19 patients before and after the development of ARDS and integrate them into the diagnostic system to achieve personalized recommendations of treatment measures.

\section{Conclusion}

We retrospectively analyzed the clinical characteristics of COVD-19 patients with and without ARDS from Zhejiang Province and Wuhan and identified 19 risk factors. Further, based on these risk factors, we used five methods for modeling, four of which had good predicting effect. The decision tree performed best with an accuracy rate of $97 \%$. We have deployed it to the infectious disease electronic medical record system to assist doctors in early warning severe patients with COVID-19.

\section{Method}

\subsection{Patient Population and Clinical Data}

Data on a total of 659 consecutive COVID-19 patients from January 22 to April 1, 2020 were retrospectively collected in hospitals from 11 regions $₫$ NingBo, ZhouShan, HuBei, Lishui, Jiaxin, HangZhou, TaiZhou, DongYang, ShaoXing, WenZhou. The age of the patient ranged from 14 and 90 years old. All patients were diagnosed by positive tests of severe acute respiratory syndrome-coronavirus2(SARS-CoV-2) nucleic acids, according to WHO interim guidance. Clinical information including demographic, comorbidities, epidemiological history of exposure to COVID-19, vital sign, clinical symptoms, biochemical indices, blood routine, infection-related biomarkers, CT findings, therapeutic measures, and all the time information from onset to admission were collected from routine clinical practice. The date of disease onset was defined as the day when symptoms (i.e. fever, dry cough, expectoration, polypnea, fatigue, myalgia, pharyngalgia, dyspnea, headache, vomiting) first appeared. 
ARDS was defined according to the Berlin definition. Severity evaluation criteria on admission was based on the Guidelines for the Diagnosis and Treatment of Novel Coronavirus (2019-nCoV) Infection (Trial Version 7), which was a comprehensive evaluation index with important clinical diagnostic value. Patients with one of the following symptoms are diagnosed as secondary bacterial infection: bacteria are found in sterile sites; patients have a fever that was unrelated to the initial disease, accompanied by elevated CRP. This study was approved by the Ethics Committee of Shulan Hangzhou Hospital. Written informed consents were signed during hospitalization from patients or their parents.

\subsection{Data Analysis}

Continuous variables were expressed as medians and interquartile ranges or simple ranges, as defined by experts. Categorical variables were summarized as counts and percentages. We assessed differences between ARDS and non-ARDS using Two-Sample T test or Mann-Whitney $U$ test depending on parametric or non-parametric data for continuous variables and the Chi-square for categorical variables. Tests were two-sided with significance set at a less than 0.05. All statistical analysis was performed using IBM SPSS Ver. 19.0. The Python programming language (Python Software Foundation, version 3.6.6冈https://www.python.org/downloads/) was used for our models.

\subsection{Machine Learning Model Establishment and Evaluation}

Datasets: All data was divided into three separate parts with no overlapping topics: training, validation, and external test sets (Table 7).

For COVID-19 ARDS prediction

- Training and validation datasets: 236 subjects were assigned to the training and validation datasets following a 9:1 ratio, including 189 non-ARDS and 47 ARDS cases from 11 regions in Wuhan and Zhejiang, further cross-validated 10 times. These datasets were used to train model parameters.

- External test dataset: There were 57 non-ARDS and 14 ARDS cases from 11 regions in Wuhan and Zhejiang. This dataset was used to evaluate and analyze the performances of different models to

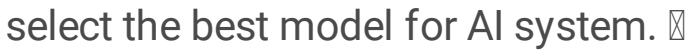

Algorithms: Four conventional types of machine learning algorithms (decision trees, random forests, support vector machines and logistic regression) and one deep learning method with ReLu activation function (deep neural networks $₫ D N N$ ) were conducted to develop the ARDS prediction model in COVID-19 patients. We implemented support vector machines with the RBF kernel. ID3 decision tree was constructed with the max leaf nodes of 5 and random forest was constructed by 40 decision trees with criterion of entropy algorithm. The pipeline of the DNN model was shown in Figure S1. The input data was a 19-dimensional vector, containing the clinical data of patients. The DNN model employed in this study was a 4-layer network structure with the hidden neurons of $64,32,8$ and 1 respectively. A sigmoid 
layer was added at the top of the network to output the probability of ARDS occurrence and a total of 100 epochs were executed.

Evaluation: The performance of the models was assessed by 10 -fold cross validation (10-fold CV) and external tests. Specifically, we randomly divided the training and validation datasets into 10 parts: 9 parts were used to train the algorithms and 1 part was used to estimate the prediction performance of the method. The mean AUC and accuracy were calculated by 10 -fold CV as indicators of prediction accuracy. This process was repeated 10 times. Furthermore, we verify the prediction accuracy of the models on the external test dataset by evaluating the receiver operating characteristic (ROC) curves, the classification accuracy, F-measure, sensitivity and specificity.

\subsection{Application Development}

The best algorithm for ARDS risk prediction was embedded into EMR and could be accessed via the link https://ai-ards.rubikstack.com/\#/login. The Anaconda Distribution (Anaconda Inc, Austin, Texas), Visual Studio Code version 1.45.1 (Microsoft, Redmond, Washington), and Python version 3.6 (Python Software Foundation, Wilmington, Delaware) were used for data analysis, model creation, and web application development.

\section{Declarations}

\section{Acknowledgments}

We would like to thank the medical staffs in the Department of Infectious Disease for their support in providing information about patients. We also thank the patients for their willingness to participate. Finally, we thank Prof. Peilin Yu and Dr. Lin Wei for their generous help. This work was supported by COVID-19 Emergency Research Project of Zhejiang Provincial Department of Science and Technology (2020C03123) and National Science and Technology Major Project (No.2017ZX10204401).

\section{Author contributions}

W.X. wrote the main manuscript text and prepared tables 1-4. N.N.S. and W.X. prepared tables5-7 and all figures. H.N.G. provided original dataset. Z.Y.C. and Y.Y. modified the main manuscript. B.J. and L.L.T. were the corresponding author of the article and guided the research. All authors reviewed the manuscript.

\section{Additional Information}

\section{- Ethics approval and consent to participate}

This study has been approved by the ethics committee of ShuLan (Hangzhou) Hospital. All procedures performed in studies involving human participants were in accordance with the ethical standards of the institutional and/or national research committee and with the 1964 Helsinki declaration and its later 
amendments or comparable ethical standards. Written informed consents were signed during hospitalization from patients or their parents. The data used in this study were anonymized before its use.

\section{- Conflict of Interest Statement}

The authors declare that they have no conflict of interest.

\section{- Source of Funding}

The results reported herein correspond to specific aims of grant 2020C03123 to investigator Ling-Ling Tang from COVID-19 Emergency Research Project of Zhejiang Provincial Department of Science and Technology. This work was also supported by grant No.2017ZX10204401 from National Science and Technology Major Project.

\section{References}

1. Guan WJ, Ni ZY, Hu Y, Liang WH, Ou CQ, He JX, et al. Clinical Characteristics of Coronavirus Disease 2019 in China. N Engl J Med. 2020;382(18):1708-1720.

2. Huang C, Wang Y, Li X, Ren L, Zhao J, Hu Y, et al. Clinical features of patients infected with 2019 novel coronavirus in Wuhan, China. The Lancet. 2020;395(10223):497-506.

3. Ding Q, Lu P, Fan Y, Xia Y, Liu M. The clinical characteristics of pneumonia patients coinfected with 2019 novel coronavirus and influenza virus in Wuhan, China. Journal of Medical Virology. 2020.

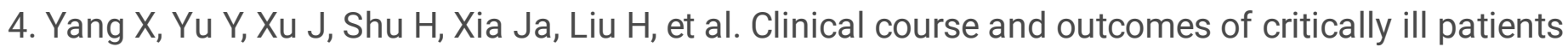
with SARS-CoV-2 pneumonia in Wuhan, China: a single-centered, retrospective, observational study. The Lancet Respiratory Medicine. 2020;8(5):475-481.

5. Liu W, Tao Z-W, Wang L, Yuan M-L, Liu K, Zhou L, et al. Analysis of factors associated with disease outcomes in hospitalized patients with 2019 novel coronavirus disease. Chinese Medical Journal. 2020;133(9):1032-1038.

6. Zhao X-Y, Xu X-X, Yin H-S, Hu Q-M, Xiong T, Tang Y-Y, et al. Clinical characteristics of patients with 2019 coronavirus disease in a non-Wuhan area of Hubei Province, China: a retrospective study. BMC Infectious Diseases. 2020;20(1):311.

7. Li K, Wu J, Wu F, Guo D, Chen L, Fang Z, et al. The Clinical and Chest CT Features Associated with Severe and Critical COVID-19 Pneumonia. Investigative Radiology. 2020;55(6):327-331.

8. Bellani G, Laffey JG, Pham T, Fan E, Brochard L, Esteban A, et al. Epidemiology, Patterns of Care, and Mortality for Patients with Acute Respiratory Distress Syndrome in Intensive Care Units in 50 Countries. JAMA. 2016;315(8):788-800.

9. Yang $X, Y u$ Y, Xu J, Shu H, Xia J, Liu H, et al. Clinical course and outcomes of critically ill patients with SARS-CoV-2 pneumonia in Wuhan, China: a single-centered, retrospective, observational study. Lancet Respir Med. 2020;8(5):475-481. 
10. Jiang X, Coffee M, Bari A, Wang J, Jiang X, Huang J, et al. Towards an Artificial Intelligence Framework for Data-Driven Prediction of Coronavirus Clinical Severity. Computers, Materials \& Continua. 2020;62(3):537-551.

11. Shortliffe EH, Sepulveda MJ. Clinical Decision Support in the Era of Artificial Intelligence. JAMA. 2018;320(21):2199-200.

12. Wang S, Zha Y, Li W, Wu Q, Li X, Niu M, et al. A Fully Automatic Deep Learning System for COVID-19 Diagnostic and Prognostic Analysis. Eur Respir J. 2020.

13. Gong J, Ou J, Qiu X, Jie Y, Chen Y, Yuan L, et al. A Tool to Early Predict Severe Corona Virus Disease 2019 (COVID-19): A Multicenter Study using the Risk Nomogram in Wuhan and Guangdong, China. Clin Infect Dis. 2020. doi: 10.1183/13993003.00775-2020.

14. Meylan S, Akrour R, Regina J, Bart PA, Dami F, Calandra T. An Early Warning Score to predict ICU admission in COVID-19 positive patients. J Infect. 2020. doi: 10.1016/j.jinf.2020.05.047

15. Yang Z, Zeng Z, Wang K, Wong SS, Liang W, Zanin M, et al. Modified SEIR and Al prediction of the epidemics trend of COVID-19 in China under public health interventions. J Thorac Dis. 2020;12(3):165-174.

16. Bai X, Fang C, Zhou Y, Bai S, Liu Z, Chen Q, et al. Predicting COVID-19 malignant progression with AI techniques. medRxiv. 2020:2020.03.20.20037325.

17. Wynants L, Van Calster B, Bonten MMJ, Collins GS, Debray TPA, De Vos M, et al. Prediction models for diagnosis and prognosis of covid-19 infection: systematic review and critical appraisal. BMJ. 2020;369:m1328.

18. Zheng Z, Peng F, Xu B, Zhao J, Liu H, Peng J, et al. Risk factors of critical \& mortal COVID-19 cases: A systematic literature review and meta-analysis. J Infect. 2020, 81: e16-e25

19. Wu C, Chen X, Cai Y, Xia J, Zhou X, Xu S, et al. Risk Factors Associated with Acute Respiratory Distress Syndrome and Death in Patients with Coronavirus Disease 2019 Pneumonia in Wuhan, China. JAMA Intern Med. 2020. doi: 10.1001/jamainternmed.2020.0994

20. Liang W, Liang H, Ou L, Chen B, Chen A, Li C, et al. Development and Validation of a Clinical Risk Score to Predict the Occurrence of Critical Illness in Hospitalized Patients With COVID-19. JAMA Intern Med. 2020 Aug 1;180(8):1081-1089. doi: 10.1001/jamainternmed.2020.2033

21. Mirzaei R, Goodarzi P, Asadi M, Soltani A, Aljanabi HAA, Jeda AS, et al. Bacterial co-infections with SARS-CoV-2. IUBMB Life. 2020. doi: 10.1002/iub.2356

22. Manna S, Baindara $P$, Mandal SM. Molecular pathogenesis of secondary bacterial infection associated to viral infections including SARS-CoV-2. J Infect Public Health. 2020. doi: 10.1016/j.jiph.2020.07.00

23. Agassandian M, Shurin GV, Ma Y, Shurin MR. C-reactive protein and lung diseases. Int J Biochem Cell Biol. 2014; 53:77-88.

24. Liu Y, Yang Y, Zhang C, Huang F, Wang F, Yuan J, et al. Clinical and biochemical indexes from 2019nCoV infected patients linked to viral loads and lung injury. Sci China Life Sci. 2020;63(3):364-74. 
25. Li X, Wang L, Yan S, Yang F, Xiang L, Zhu J, et al. Clinical characteristics of 25 death cases with COVID-19: A retrospective review of medical records in a single medical center, Wuhan, China. Int $J$ Infect Dis. 2020; 94:128-32.

26. Li X, Xu S, Yu M, Wang K, Tao Y, Zhou Y, et al. Risk factors for severity and mortality in adult COVID19 inpatients in Wuhan. J Allergy Clin Immunol. 2020. doi: 10.1016/j.jaci.2020.04.006

27. Cai Q, Huang D, Yu H, Zhu Z, Xia Z, Su Y, et al. COVID-19: Abnormal liver function tests. J Hepatol. 2020. doi: 10.1016/j.jhep.2020.04.006

28. Fan Z, Chen L, Li J, Cheng X, Yang J, Tian C, et al. Clinical Features of COVID-19-Related Liver Functional Abnormality. Clin Gastroenterol Hepatol. 2020;18(7):1561-6.

29. Guthrie GJ, Charles KA, Roxburgh CS, Horgan PG, McMillan DC, Clarke SJ. The systemic inflammation-based neutrophil-lymphocyte ratio: experience in patients with cancer. Crit Rev Oncol Hematol. 2013;88(1):218-30.

30. Shimoyama Y, Umegaki O, Inoue S, Agui T, Kadono N, Minami T. The Neutrophil to Lymphocyte Ratio Is Superior to Other Inflammation-Based Prognostic Scores in Predicting the Mortality of Patients with Pneumonia. Acta Med Okayama. 2018;72(6):591-3.

31. Jeon Y, Lee WI, Kang SY, Kim MH. Neutrophil-to-Monocyte-Plus-Lymphocyte Ratio as a Potential Marker for Discriminating Pulmonary Tuberculosis from Nontuberculosis Infectious Lung Diseases. Lab Med. 2019;50(3):286-91.

32. Ying HQ, Deng QW, He BS, Pan YQ, Wang F, Sun HL, et al. The prognostic value of preoperative NLR, d-NLR, PLR and LMR for predicting clinical outcome in surgical colorectal cancer patients. Med Oncol. 2014;31(12):305.

33. Uslu AU, Kucuk A, Sahin A, Ugan Y, Yilmaz R, Gungor T, et al. Two new inflammatory markers associated with Disease Activity Score-28 in patients with rheumatoid arthritis: neutrophillymphocyte ratio and platelet-lymphocyte ratio. Int J Rheum Dis. 2015;18(7):731-735.

34. Yang AP, Li HM, Tao WQ, Yang XJ, Wang M, Yang WJ, et al. Infection with SARS-CoV-2 causes abnormal laboratory results of multiple organs in patients. Aging (Albany NY). 2020;12. doi.org/10.18632/aging.103255

35. Shi S, Qin M, Shen B, Cai Y, Liu T, Yang F, et al. Association of Cardiac Injury with Mortality in Hospitalized Patients With COVID-19 in Wuhan, China. JAMA Cardiol. 2020. doi:10.1001/jamacardio.2020.0950

36. Chan KH, Farouji I, Abu Hanoud A, Slim J. Weakness and elevated creatinine kinase as the initial presentation of coronavirus disease 2019 (COVID-19). Am J Emerg Med. 2020. doi: 10.1016/j.ajem.2020.05.015

37. Cabral BMI, Edding SN, Portocarrero JP, Lerma EV. Rhabdomyolysis. Dis Mon. 2020:101015.

38. Suwanwongse K, Shabarek N. Rhabdomyolysis as a Presentation of 2019 Novel Coronavirus Disease. Cureus. 2020;12(4): e7561.

39. Jin M, Tong Q. Rhabdomyolysis as Potential Late Complication Associated with COVID-19. Emerg Infect Dis. 2020;26(7). 
40. Gefen AM, Palumbo N, Nathan SK, Singer PS, Castellanos-Reyes LJ, Sethna CB. Pediatric COVID-19associated rhabdomyolysis: a case report. Pediatr Nephrol. 2020. doi:10.1007/s00467-020-04617-0

41. Kourou K, Exarchos TP, Exarchos KP, Karamouzis MV, Fotiadis DI. Machine learning applications in cancer prognosis and prediction. Comput Struct Biotechnol J. 2015; 13:8-17.

42. Visweswaran S, Ferreira A, Ribeiro GA, Oliveira AC, Cooper GF. Personalized Modeling for Prediction with Decision-Path Models. PLoS One. 2015;10(6): e0131022.

43. Che D, Liu Q, Rasheed K, Tao X. Decision tree and ensemble learning algorithms with their applications in bioinformatics. Adv Exp Med Biol. 2011; 696:191-9.

\section{Tables}


Table 1 Demographic and Epidemiology of the Study Patients

\begin{tabular}{llll}
\hline Characteristics & Patients & ARDS & Non-ARDS Patients \\
$(\mathrm{N}=659)$ & $\begin{array}{l}\mathrm{P} \\
\text { Patients } \\
(\mathrm{N}=76)\end{array}$ & $\begin{array}{c}(\mathrm{N}=583) \\
\text { value }\end{array}$ \\
\hline
\end{tabular}

\section{Demography}

Age

Median(IQR)- years

$\begin{array}{lll}50.0(37.0-62.0) & \begin{array}{l}56.5(47.5- \\ 67.8)\end{array} & 49.0(36.0-60.0) \\ & & \\ & & \\ 70 / 659(10.6 \%) & 2 / 76(2.6 \%) & 68 / 583(11.7 \%) \\ 545 / 659(82.7 \%) & 61 / 76(80.3 \%) & 484 / 583(83 \%) \\ 44 / 659(6.7 \%) & 13 / 76(17.1 \%) & 31 / 583(5.3 \%)\end{array}$

Gender

$\begin{array}{lll}332 / 659(50.4 \%) & 58 / 76(76.3 \%) & 274 / 583(47.0 \%) \\ 327 / 659(49.6 \%) & 18 / 76(23.7 \%) & 309 / 583(53.0 \%)\end{array}$

Female - no./total no. (\%)

$327 / 659(49.6 \%)$

18/76(23.7\%) 309/583(53.0\%)

BMI

Median(IQR)

$23.9(21.5-25.9)$

25.5(21.5-

23.7(23.2-27.7)

0.001

Distribution - no./total no. (\%) 25.7)

0.000

$<23$

229/591 (38.7\%)

$14 / 65(21.5 \%)$

$215 / 526(40.9 \%)$

23-25

161/591(27.2\%)

17/65(26.2\%)

$144 / 526(27.4 \%)$

$>25$

201/591(34.1\%)

34/65(52.3\%) 167/526(31.7\%)

Medical staff - no./total no. (\%)

$9 / 659(1.4 \%)$

$1 / 76(1.3 \%)$

8/583(1.4\%)

0.968

$11 / 659(1.67 \%)$

$3 / 76(3.9 \%)$

8/583(1.4\%)

0.123

$51 / 654(7.8 \%)$

$11 / 76(14.5 \%)$

$40 / 578(6.9 \%)$

0.198

Epidemiology - no./total no. (\%)

History of exposure

$\begin{array}{llll}447 / 630(70.9 \%) & 38 / 70(54.3 \%) & 409 / 560(73.0 \%) & 0.002 \\ 258 / 583(44.3 \%) & 27 / 76(35.5 \%) & 231 / 507(45.6 \%) & 0.285 \\ 1(0-13) & 1(0-1) & 1(0-1) & 0.263 \\ 381 / 657(57.9 \%) & 34 / 76(44.7 \%) & 347 / 581(59.7 \%) & 0.091 \\ 33 / 608(5.4 \%) & 33 / 73(45.2 \%) & 0 / 535(0 \%) & 0.000 \\ 5(3-9) & 6(3-8) & 5(3-10) & 0.901\end{array}$

Interval between date of contact and date of onset -

Median (IQR)

The interval between the onset date and the visit date

Median (IQR)

$2(0-5)$

$3(1-6)$

$1(0-4)$

0.772 
$\leq 2$ days

3-5 days

$>5$ days

The interval between the date of onset and the date of admission

Median (IQR)

Distribution - no./total no. (\%)

$\leq 2$ days

3-5 days

$>5$ days

The interval between the onset date and the antiviral date

Median (IQR)

Distribution - no./total no. (\%)

$\leq 2$ days

3-5 days

$>5$ days
385/651(59.1\%)

$34 / 75(45.3 \%)$

$351 / 576(60.9 \%)$

$142 / 651(21.8 \%)$

$22 / 75(29.3 \%)$

$120 / 576(20.8 \%)$

$124 / 651(19.0 \%)$

$19 / 75(25.3 \%)$

105/576(18.3\%)

5(2-10)

6(3-8)

$5(3-8)$

0.759

0.250

$\begin{array}{lll}182 / 656(27.7 \%) & 15 / 76(19.7 \%) & 167 / 580(28.8 \%) \\ 167 / 656(25.5 \%) & 22 / 76(28.9 \%) & 145 / 580(25.0 \%) \\ 307 / 656(46.8 \%) & 39 / 76(51.3 \%) & 268 / 580(46.2 \%)\end{array}$

5(3-10)

4(4-9)

5(4-9)

0.278

0.250

$\begin{array}{lll}182 / 656(27.7 \%) & 15 / 76(19.7 \%) & 167 / 580(28.8 \%) \\ 167 / 656(25.5 \%) & 22 / 76(28.9 \%) & 145 / 580(25.0 \%) \\ 307 / 656(46.8 \%) & 39 / 76(51.4 \%) & 268 / 580(46.2 \%)\end{array}$

Abbreviations: BMI, body mass index; ICU, intensive care unit; no., number Data are presented as medians (interquartile ranges, IQR) \and no./total no. (\%) 
Table 2 Clinical Characteristics and Underlying Diseases

\begin{tabular}{|c|c|c|c|c|}
\hline Characteristics & $\begin{array}{l}\text { Patients } \\
(\mathrm{N}=659)\end{array}$ & $\begin{array}{l}\text { ARDS Patients } \\
(\mathrm{N}=76)\end{array}$ & Non-ARDS Patients $(\mathrm{N}=583)$ & P value \\
\hline \multicolumn{5}{|l|}{ Clinical symptoms - no./total no. (\%) } \\
\hline Severity evaluation at admission & & & & 0.000 \\
\hline mild & $59 / 643(9.2 \%)$ & $0 / 76(0.0 \%)$ & $59 / 567(10.4 \%)$ & \\
\hline severe & $67 / 643(10.4 \%)$ & $29 / 76(38.2 \%)$ & $38 / 567(6.7 \%)$ & \\
\hline critical & $32 / 643(5.0 \%)$ & $32 / 76(42.1 \%)$ & $0 / 567(0.0 \%)$ & \\
\hline Median (IQR) $-{ }^{\circ} \mathrm{C}$ & $37.5(36.8-38.2)$ & $37.9(37.3-38.5)$ & $37.4(36.8-38.1)$ & 0.000 \\
\hline Distribution - no./total no. (\%) & & & & 0.000 \\
\hline$\square 37.3^{\circ} \mathrm{C}$ & $275 / 659(41.7 \%)$ & $18 / 76(23.7 \%)$ & $257 / 583(44.1 \%)$ & \\
\hline $37.3-38^{\circ} \mathrm{C}$ & $198 / 659(30.0 \%)$ & $24 / 76(31.6 \%)$ & $174 / 583(29.8 \%)$ & \\
\hline $38.1-39^{\circ} \mathrm{C}$ & $153 / 659(23.2 \%)$ & $24 / 76(31.6 \%)$ & $129 / 583(22.1 \%)$ & \\
\hline$>39^{\circ} \mathrm{C}$ & $33 / 659(5.0 \%)$ & $10 / 76(13.2 \%)$ & $23 / 583(3.9 \%)$ & \\
\hline Yellow sputum & $32 / 643(5.0 \%)$ & $3 / 73(4.1 \%)$ & $29 / 570(5.1 \%)$ & 0.049 \\
\hline Hemoptysis & $10 / 626(1.6 \%)$ & $4 / 73(5.5 \%)$ & $6 / 553(1.1 \%)$ & 0.017 \\
\hline Sore throat & $81 / 635(12.8 \%)$ & $9 / 72(12.5 \%)$ & $72 / 563(12.8 \%)$ & 0.051 \\
\hline Stuffy nose & $24 / 631(3.8 \%)$ & $1 / 72(1.4 \%)$ & $23 / 559(4.1 \%)$ & 0.506 \\
\hline Muscle ache & 108/633(17.1\%) & $7 / 72(9.7 \%)$ & $101 / 561(18.0 \%)$ & 0.095 \\
\hline Fatigue & $221 / 646(34.2 \%)$ & $27 / 73(36.9 \%)$ & 194/573(33.9\%) & 0.366 \\
\hline Shortness of breath & $110 / 635(17.3 \%)$ & $45 / 76(59.2 \%)$ & $65 / 559(11.6 \%)$ & 0.000 \\
\hline Gastrointestinal symptoms & $121 / 645(18.8 \%)$ & $14 / 74(18.9 \%)$ & $107 / 571(18.7 \%)$ & 0.948 \\
\hline Diarrhea & $91 / 607(15.0 \%)$ & $15 / 73(20.5 \%)$ & $76 / 534(14.2 \%)$ & 0.179 \\
\hline Vomiting & $18 / 603(3.0 \%)$ & $5 / 73(6.8 \%)$ & $13 / 530(2.4 \%)$ & 0.054 \\
\hline Nausea & $60 / 609(9.9 \%)$ & $5 / 73(6.8 \%)$ & $55 / 536(10.3 \%)$ & 0.323 \\
\hline
\end{tabular}


Underlying diseases - no./total no. (\%)

\begin{tabular}{|c|c|c|c|c|}
\hline Basic disease & $274 / 657(41.7 \%)$ & $43 / 76(56.6 \%)$ & $231 / 581(39.8 \%)$ & 0.006 \\
\hline Diabetes & $66 / 628(10.5 \%)$ & $13 / 73(17.8 \%)$ & $53 / 555(9.5 \%)$ & 0.041 \\
\hline COPD & $7 / 614(1.1 \%)$ & $1 / 73(1.4 \%)$ & $6 / 541(1.1 \%)$ & 0.590 \\
\hline Asthma & $3 / 614(0.5 \%)$ & $0 / 73(0.0 \%)$ & $3 / 541(0.6 \%)$ & 0.524 \\
\hline Malignancy & $9 / 629(1.4 \%)$ & $1 / 73(1.4 \%)$ & $8 / 556(1.4 \%)$ & 0.971 \\
\hline Blood system diseases & $2 / 613(0.3 \%)$ & $1 / 73(1.4 \%)$ & $1 / 540(0.2 \%)$ & 0.096 \\
\hline Chronic hepatopath & $29 / 631(4.6 \%)$ & $5 / 73(6.8 \%)$ & $24 / 558(4.3 \%)$ & 0.328 \\
\hline Chronic nephrosis & $6 / 616(1.0 \%)$ & $1 / 73(1.4 \%)$ & $5 / 543(0.9 \%)$ & 0.756 \\
\hline Pneumonia on admission & $591 / 647(91.3 \%)$ & $75 / 76(98.7 \%)$ & $516 / 571(90.4 \%)$ & 0.056 \\
\hline Acute kidney injury on admission & $9 / 652(1.4 \%)$ & $3 / 76(3.9 \%)$ & $6 / 576(1.0 \%)$ & 0.076 \\
\hline With other respiratory virus infections & $5 / 569(0.9 \%)$ & $2 / 73(2.7 \%)$ & $3 / 496(0.6 \%)$ & 0.126 \\
\hline
\end{tabular}

Abbreviations: COPD, chronic obstructive pulmonary disease; no., number

Data are presented as medians (interquartile ranges, IQR) Land no./total no. (\%) 
Table 3 Radiologic, Laboratory Findings and Complications

\begin{tabular}{|c|c|c|c|c|}
\hline Variables & $\begin{array}{l}\text { Patients } \\
(\mathrm{N}=659)\end{array}$ & $\begin{array}{l}\text { ARDS Patients } \\
(\mathrm{N}=76)\end{array}$ & $\begin{array}{c}\text { Non-ARDS Patients } \\
(\mathrm{N}=583)\end{array}$ & $\begin{array}{l}P \\
\text { value }\end{array}$ \\
\hline \multicolumn{5}{|l|}{ CT image features - no./total no. (\%) } \\
\hline Consolidation & $175 / 619(28.3 \%)$ & $41 / 76(53.9 \%)$ & $134 / 543(24.7 \%)$ & 0.000 \\
\hline Ground-glass opacity & $467 / 625(74.7 \%)$ & $59 / 73(80.8 \%)$ & $408 / 552(73.9 \%)$ & 0.251 \\
\hline Number of Consolidation quadrant - Median (IQR) & $0(0-2)$ & $2(1-4)$ & $0(0-2)$ & 0.000 \\
\hline \multicolumn{5}{|l|}{ Laboratory findings } \\
\hline \multicolumn{5}{|l|}{ Leukocyte count within 48 hours of admission (10e9/l) } \\
\hline Median (IQR) & $5.0(4.0-6.6)$ & $5.7(4.6-8.8)$ & $4.9(3.9-6.4)$ & 0.000 \\
\hline Distribution - no./total no. (\%) & & & & 0.000 \\
\hline$\square 4$ & $163 / 657(24.8 \%)$ & $14 / 76(18.4 \%)$ & 149/581(25.6\%) & \\
\hline $4-10$ & $468 / 657(71.2 \%)$ & $47 / 76(61.8 \%)$ & $421 / 581(72.5 \%)$ & \\
\hline$>10$ & $26 / 657(4.0 \%)$ & $15 / 76(19.7 \%)$ & $11 / 581(1.9 \%)$ & \\
\hline Neutrophil count (10e9/l) -Median(IQR) & $3.2(2.3-4.4)$ & $4.8(3.1-7.6)$ & $3.1(2.3-4.2)$ & 0.000 \\
\hline \multicolumn{5}{|l|}{ Lymphocyte count (10e9/l) } \\
\hline Median (IQR) & $1.1(0.8-1.6)$ & $0.7(0.5-1.1)$ & $1.2(0.8-1.7)$ & 0.000 \\
\hline Distribution - no./total no. (\%) & & & & 0.000 \\
\hline$\leq 1.5$ & $468 / 659(71.0 \%)$ & $71 / 76(93.4 \%)$ & $397 / 583(68.1 \%)$ & \\
\hline$>1.5$ & 191/659(29.0\%) & $5 / 76(6.6 \%)$ & 186/583(31.9\%) & \\
\hline \multicolumn{5}{|l|}{ Lymph\% } \\
\hline Median (IQR) & $0.24(0.2-0.3)$ & $0.13(0.1-0.2)$ & $0.25(0.2-0.3)$ & 0.403 \\
\hline \multicolumn{5}{|l|}{ Distribution - no./total no. (\%) } \\
\hline$<0.2$ & $237 / 657(36.1 \%)$ & $56 / 76(73.7 \%)$ & $181 / 581(31.2 \%)$ & 0.000 \\
\hline$>=0.2$ & $420 / 657(63.9 \%)$ & $20 / 76(26.3 \%)$ & $400 / 581(68.8 \%)$ & \\
\hline \multicolumn{5}{|l|}{ Neutrophils / lymphocytes } \\
\hline Median (IQR) & $2.73(1.8-4.5)$ & $6.11(3.7-14.6)$ & $1.2(1.7-4.0)$ & 0.000 \\
\hline Distribution - no./total no. (\%) & & & & 0.000 \\
\hline$<3$ & $348 / 636(54.7 \%)$ & $13 / 75(17.3 \%)$ & $335 / 561(59.7 \%)$ & \\
\hline$\geq 3$ & 288/636(45.3\%) & $62 / 75(82.7 \%)$ & $226 / 561(40.3 \%)$ & \\
\hline Hemoglobin (g/l)- Median (IQR) & $\begin{array}{l}134.0(123.0- \\
146.0)\end{array}$ & $\begin{array}{l}135.5(123.3- \\
150.0)\end{array}$ & 134(123.0-144.0) & 0.462 \\
\hline Hematocrit (\%)- Median (IQR) & $0.4(0.0-0.4)$ & $0.4(0.3-0.4)$ & $0.39(0.4-0.5)$ & 0.968 \\
\hline
\end{tabular}


Platelet (10e9/l)

Median (IQR)

Distribution - no./total no. (\%)

$\leq 100$

$>100$

Alanine aminotransferase (U/L)

Median (IQR)

Distribution - no./total no. (\%)

$\leq 40$

$>40$

Aspartate aminotransferase (U/L)

Median (IQR)

Distribution - no./total no. (\%)

$\leq 40$

$>40$

Potassium ion (mmol/L) - Median(IQR)

Sodium ion (mmol/L) - Median(IQR)

Creatinine (umol/l)

Median (IQR)

Distribution - no./total no. (\%)

$\leq 133$

$>133$

Creatine Kinase (U/L)

Median (IQR)

Distribution - no./total no. (\%)

$<=185$

$>185$

Lactate dehydrogenase (U/L)

Median (IQR)

Distribution - no./total no. (\%)

$<=250$

$>250$
$24.0(18.0-31.0)$

$31.5(24.0-46.0)$

$23(18.0-30.0)$

0.000

$\begin{array}{lll}24 / 658(3.6 \%) & 4 / 76(5.3 \%) & 20 / 582(3.4 \%) \\ 634 / 658(96.4 \%) & 72 / 76(94.7 \%) & 562 / 582(96.6 \%)\end{array}$

$21.0(15-34.0)$

$30.3(21.3-46.0)$

$20.0(14.0-32.0)$

0.000

0.000

$\begin{array}{lll}533 / 654(81.5 \%) & 48 / 74(64.9 \%) & 485 / 580(83.6 \%) \\ 121 / 654(18.5 \%) & 26 / 74(35.1 \%) & 95 / 580(16.4 \%)\end{array}$

0.000

$563 / 653(86.2 \%) \quad 44 / 74(59.5 \%) \quad 519 / 579(89.6 \%)$

$90 / 653(13.8 \%) \quad 30 / 74(40.5 \%) \quad 60 / 579(10.4 \%)$

$3.8(3.6-4.2) \quad 3.8(3.5-4.1) \quad 3.9(3.6-4.2)$

0.188

$138.8(136.7-$

$138.0(135.0-$

$139.0(137.0-140.7)$

0.052

146.7)

140.6)

65.3(54.0-78.0)

70.5(59.5-92.0)

65.0(53.4-77.4)

0.002

0.072

$\begin{array}{lll}648 / 657(98.6 \%) & 72 / 75(96.0 \%) & 576 / 582(99.0 \%) \\ 9 / 657(1.4 \%) & 3 / 75(4.0 \%) & 6 / 582(1.0 \%)\end{array}$

$\begin{array}{ll}74.0(50.8-107.2) & 112.5(63.0-\quad 70.6(49.0-96.3) \\ & 245.0)\end{array}$

0.000

0.000

$461 / 513(89.9 \%) \quad 47 / 68(69.1 \%) \quad 414 / 445(93.0 \%)$

$52 / 513(10.1 \%) \quad 21 / 68(30.9 \%) \quad 31 / 445(7.0 \%)$

$215.0(177.0-$

$316.0(253.8-$ 394.0)

$211.0(173.5-257.5)$

0.000 275.3)

0.000

$\begin{array}{lll}431 / 636(67.8 \%) & 17 / 69(24.6 \%) & 414 / 567(73.0 \%) \\ 205 / 636(32.2 \%) & 52 / 69(75.4 \%) & 153 / 567(27.0 \%)\end{array}$

Page $19 / 23$ 
Troponin I (ng/ml - Median (IQR)

Brain Natriuretic Peptide (<300pg/ml) - Median (IQR)

Myoglobin - Median (IQR)

Glucose (mmol/L) - Median (IQR)

C-reactive protein $(\mathrm{mg} / \mathrm{L})$

Median (IQR)

Distribution - no./total no. (\%)

$<=10$

$>10$

Procalcitonin (ng/L)

Median (IQR)

Distribution - no./total no. (\%)

$<0.1$

$0.1-0.5$

$\geq 0.5$

Highest CRP within one week of admission (mg/L) Median (IQR)

Highest PCT within one week of admission (ng/L) Median (IQR)

FiO2 on the first day of admission - Median (IQR)

$\mathrm{PaO} 2$ on the first day of admission (mmHg) - Median (IQR)

PaCO2 on the first day of admission (mmHg) - Median (IQR)

Oxygenation index on the first day of admission (mmHg)

Median (IQR)

Distribution - no./total no. (\%)

$\leq 200$

$>200$

D-dimer ( $\mu \mathrm{g} / \mathrm{L})$ - Median (IQR)

Complications - no./total no. (\%)

Shock

Secondary bacterial infection

$\begin{array}{llll}0.0(0.01-0.06) & 0.0(0.00-0.03) & 0.0(0.01-0.09) & 0.013 \\ 82.0(27-144.5) & 77.0(36.0-164.0) & 82.3(26.5-139.7) & 0.566 \\ 25.1(18.5-40.6) & 85.9(43.2-205.9) & 23.6(18.2-33.1) & 0.000 \\ 5.9(5.1-7.7) & 8.1(6.6-9.9) & 5.7(5.1-7.4) & 0.000\end{array}$

$10(3.0-27.3)$

34.1(15.3-76.0)

$8.8(2.8-24.1)$

0.000

0.000

$\begin{array}{lll}341 / 648(52.6 \%) & 14 / 73(19.2 \%) & 327 / 575(56.9 \%) \\ 307 / 648(47.4 \%) & 59 / 73(80.8 \%) & 248 / 575(43.1 \%)\end{array}$

$0.1(0.0-0.1)$

$0.1(0.1-0.2)$

$0.1(0.0-0.1)$

0.008

0.195

$\begin{array}{lll}326 / 510(63.9 \%) & 37 / 68(54.4 \%) & 289 / 442(65.4 \%) \\ 164 / 510(32.2 \%) & 27 / 68(39.7 \%) & 137 / 442(31.0 \%) \\ 20 / 510(3.9 \%) & 4 / 68(5.9 \%) & 16 / 442(3.6 \%)\end{array}$

15.5(4.0-39.6)

45.8(17.7-83.1)

$12.2(3.8-34.2)$

0.000

$0.1(0.0-0.1)$

$0.1(0.1-0.2)$

$0.1(0.0-0.1)$

0.000

$0.3(0.2-0.3)$

$0.2(0.3-0.5)$

$0.3(0.2-0.3)$

0.000

90.7(78.0-111.0

79.0(67.9-95.2)

94.0(80.2-117.0)

0.019

$37.5(34.0-41.5)$

33.0(32.4-37.5)

$38.0(34.8-41.9)$

0.699
292.2(144.4-

424.3)

$106 / 325(32.6 \%)$
$219 / 325(67.4 \%)$
$250.0(140.0-$
$525.3)$

4/647(0.6\%)

48/659(7.3\%)

4/73(5.5\%)

23/76(30.3\%) 261.2)
$352.0(64.2-466.2)$

0.000

0.000

Page 20/23 
Abbreviations: CT, computerized tomography; CRP, c-reactive protein; PCT, procalcitonin; no., number

Data are presented as medians (interquartile ranges, IQR) $\square$ and no./total no. (\%)

Table 4 Risk Factor Analysis for COVID -19

\begin{tabular}{|c|c|c|c|}
\hline Characteristics & OR & $95 \% \mathrm{CI}$ & $P$ value \\
\hline Gender (Male vs. Female) & 3.312 & $1.979-5.544$ & 0.000 \\
\hline Age $\geq 70$ years & 19.811 & $4.473-87.741$ & 0.001 \\
\hline $\mathrm{BMI}>25$ & 3.717 & $1.966-7.062$ & 0.001 \\
\hline Severity evaluation at admission & 13.206 & $8.550-20.397$ & 0.000 \\
\hline Temperature $>39{ }^{\circ} \mathrm{C}$ & 5.279 & $2.305-12.090$ & 0.000 \\
\hline Cough & 2.574 & $1.429-4.542$ & 0.002 \\
\hline Shortness of breath & 11.281 & $6.883-18.490$ & 0.000 \\
\hline Hemoptysis & 7.307 & 2.263-23.595 & 0.000 \\
\hline Hypertension & 4.105 & $2.572-6.554$ & 0.000 \\
\hline Diabetes & 2.176 & $1.161-4.078$ & 0.015 \\
\hline Secondary bacterial infection & 9.686 & $5.146-18.323$ & 0.000 \\
\hline Lung consolidation & 4.264 & $2.668-6.815$ & 0.000 \\
\hline Lymphocyte count (10e9/l) & 0.145 & $0.080-0.263$ & 0.000 \\
\hline Neutrophils / lymphocytes (<3 vs. $\geq 3$ ) & 7.211 & $3.980-13.064$ & 0.000 \\
\hline Alanine aminotransferase ( $\leq 40$ vs. $>40 \mathrm{U} / \mathrm{L}$ ) & 2.710 & $1.639-4.482$ & 0.000 \\
\hline Aspartate aminotransferase ( $\leq 40 \mathrm{vs} .>40 \mathrm{U} / \mathrm{L}$ ) & 5.139 & $3.100-8.520$ & 0.000 \\
\hline Creatine Kinase ( $\leq 185$ vs. $>185$ U/L) & 4.114 & 2.312-7.319 & 0.000 \\
\hline Lactate dehydrogenase ( $\leq 250$ vs. $>250 \mathrm{U} / \mathrm{L}$ ) & 8.104 & $4.733-13.876$ & 0.000 \\
\hline C-reactive protein ( $\leq 10 \mathrm{vs} .>10 \mathrm{mg} / \mathrm{L}$ ) & 5.959 & $3.510-10.119$ & 0.000 \\
\hline
\end{tabular}

Table 5 10-fold cross-validation results of 5 algorithms

\begin{tabular}{|c|c|c|}
\hline Models & AUC (mean \pm std.) & Accuracy (mean \pm std.) \\
\hline Decision Tree & $0.89 \pm 0.06$ & $0.88 \pm 0.06$ \\
\hline Logistic Regression & $0.86 \pm 0.07$ & $0.87 \pm 0.06$ \\
\hline Random Forest & $0.86 \pm 0.06$ & $0.85 \pm 0.06$ \\
\hline Support Vector Machine & $0.83 \pm 0.08$ & $0.81 \pm 0.05$ \\
\hline Deep Neural Networks & $0.74 \pm 0.12$ & $0.88 \pm 0.06$ \\
\hline P-value & 0.002 & 0.050 \\
\hline
\end{tabular}


Table 6 Performance of the five algorithms on external testing dataset in predicting the occurrence of COVID-19 ARDS

\begin{tabular}{lllllll}
\hline Models & AUC & Accuracy & Sensitivity & Specificity & F-measure (no-ARDS/ARDS) & Balance accuracy \\
\hline Decision Tree & 0.99 & 0.97 & 1.00 & 0.96 & $0.98 / 0.93$ & 0.98 \\
Logistic Regression & 0.98 & 0.93 & 0.93 & 0.93 & $0.95 / 0.84$ & 0.87 \\
Random Forest & 0.97 & 0.92 & 0.79 & 0.95 & $0.95 / 0.79$ & 0.83 \\
Support Vector Machine & 0.95 & 0.83 & 0.14 & 1.00 & $0.90 / 0.25$ & 0.57 \\
Deep Neural Networks & 0.95 & 0.90 & 0.71 & 0.95 & $0.94 / 0.74$ & 0.93 \\
\hline
\end{tabular}

Table 7 Details of modeling datasets

\begin{tabular}{llll}
\hline Datasets & Patients & ARDS Patients & No-ARDS Patients \\
\hline Training and validation dataset & 236 & 47 & 189 \\
External test dataset & 71 & 14 & 57 \\
\hline
\end{tabular}

\section{Figures}

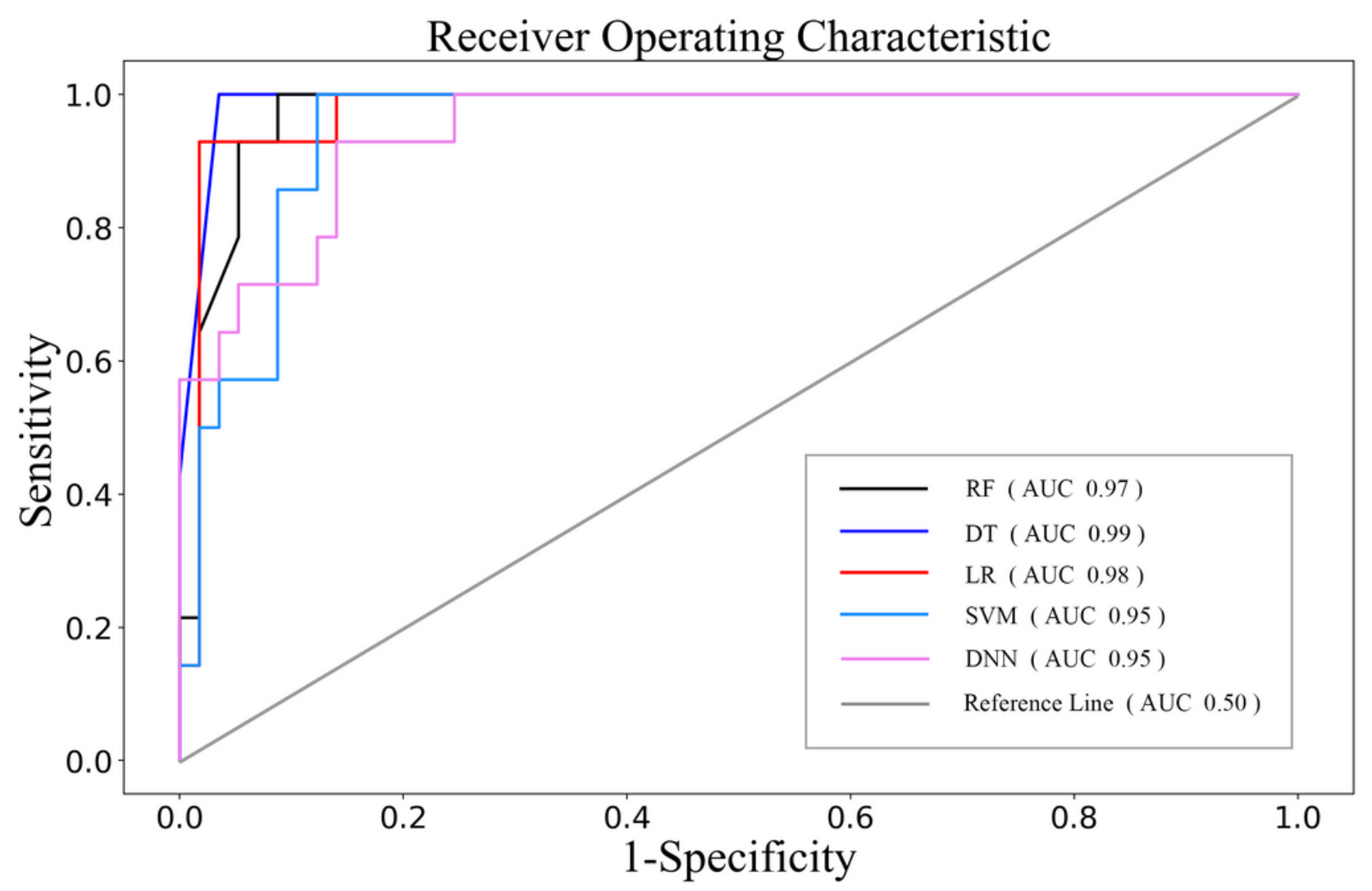

Figure 1

ROC of the five algorithms on the external testing dataset. 


\section{Supplementary Files}

This is a list of supplementary files associated with this preprint. Click to download.

- ardscode2.docx

- processeddata.xlsx

- FigureS1.tif 